\title{
The Effects of Conceptual Processing Versus Suppression on Analogue PTSD Symptoms after a Distressing Film
}

\author{
Nicole Buck \\ Maastricht University, and Erasmus Medical Center, Rotterdam, The Netherlands \\ Merel Kindt \\ University of Amsterdam, The Netherlands \\ Marcel van den Hout \\ Utrecht University, The Netherlands
}

\begin{abstract}
Background: Researchers have begun to scrutinize the assumption that active processing in response to a traumatic event is beneficial whereas avoidance of thoughts, emotions and reminders about the traumatic event is detrimental. Indications that avoidance is not always detrimental come from studies on grief and debriefing. Aims: In an analogue experimental study, the hypothesis was tested that conceptually-driven processing immediately after a distressing film is more successful in reducing analogue PTSD symptoms than suppression of thoughts and images related to the film. Method: Ninety students watched a distressing film after which they were instructed to either elaborate on the meaning of the film (conceptual processing) $(n=31)$, suppress all thoughts and images of the film by performing a task $(n=29)$, or were given no instruction $(n=30)$. Four hours later, analogue PTSD symptoms were assessed. Results: The results showed that conceptually-driven processing does not result in fewer analogue PTSD symptoms than suppression. Conclusions: It is speculated that suppression may only be dysfunctional when individuals interpret their symptoms negatively or when suppression is believed to be dysfunctional.
\end{abstract}

Keywords: PTSD, avoidance, suppression, debriefing, elaboration, conceptually-driven processing.

\section{Introduction}

Post-traumatic stress disorder (PTSD) appears to result from disturbed processing of the traumatic event. Successful processing is thought to involve expressing thoughts and feelings about the trauma so that the traumatic event can be integrated with other autobiographical

Reprint requests to Nicole Buck, Erasmus Medical Center, Department of Forensic Psychiatry, PO 2040, 3000 CA Rotterdam, The Netherlands. E-mail: n.buck@erasmusmc.nl 
memories (Ehlers and Clark, 2000), while excessive avoidance of such thoughts and feelings is hypothesized to contribute to the maintenance of PTSD symptoms after a traumatic event (Ehlers and Clark, 2000; Foa and Riggs, 1993; Horowitz, 1976; McNally, Bryant and Ehlers, 2003). Note that for most trauma victims, symptoms decline in the first months after a traumatic event without therapeutic intervention (Kessler, Sonnega, Bromet, Hughes and Nelson, 1995; Rothbaum, Foa, Riggs, Murdock and Walsh, 1992). Little is known about the strategies used by those in whom PTSD symptoms decline without therapeutic help. In principle, avoidance or suppression may have helped them to regain their lives.

There are indications that avoidance may not be as detrimental as is often held (e.g. Bonanno, Keltner, Holen and Horowitz, 1995; Bonanno, Noll, Putnam, O'Neill and Trickett, 2003), and that it is not necessarily better to confront distressing experiences than to avoid them (Stroebe and Stroebe, 1991). At least some trauma victims may in fact be helped by keeping a stiff upper lip, that is, by avoiding thoughts, emotions and reminders about the traumatic event. In line with this hypothesis are findings from debriefing studies. Debriefing that is designed to normalize reactions to the trauma and to promote the expression of feelings and thoughts connected to the trauma (Ehlers and Clark, 2003) does not prevent the development of chronic PTSD and may even be detrimental (e.g. Emmerik, Kamphuis, Hulsbosch and Emmelkamp, 2002; Mayou, Ehlers and Hobbs, 2000). Moreover, a recent study in a nationally representative sample on expressing thoughts and emotions shortly after 9/11 suggests that such expressions may even predict a worse psychological outcome (Seery, Silver, Holman, Ence and Chu, 2008). As a result, researchers have begun to question the assumption that emotional processing is beneficial, whereas avoidance is detrimental (McNally et al., 2003).

On the other hand, Ehlers and Clark (2000) argue that suppression of intrusions contributes to the development of PTSD. They argue that traumatic events should be processed conceptuallydriven. This refers to processing the meaning of the situation and putting it into context. This type of processing may benefit from detailed memories of the traumatic event. Note that trauma victims often have difficulty in intentionally retrieving parts of the traumatic event, whereas intrusive memories are very detailed and vivid (Ehlers and Clark, 2000), and thus may provide information about what happened during the event. Therefore, it might be suggested that intrusions should not be suppressed. In line with this hypothesis, several studies have found that suppression of intrusions is predictive of the maintenance of PTSD symptoms (e.g. Ehring, Ehlers and Glucksman, 2006; Mayou et al., 2000).

As noted above, Seery et al. (2008) showed that those who did not express their feelings shortly after 9/11 reported less PTSD symptoms over the following 2 years compared to those who actually expressed their feelings. Expressing one's feelings shortly after a distressing event may result from more trauma-related distress, and may thus be a vulnerability factor. The authors concluded that choosing not to express in the wake of a trauma might reflect resilience. However, this study remains silent on the supposed causal relation between suppression and PTSD symptoms. The present study aims to test the hypothesis that suppression does not cause more PTSD symptoms than conceptually-driven processing. For ethical reasons, we tested this hypothesis in an analogue study.

In the last decade, the trauma film paradigm has proven to be a useful analogue to real life trauma. Trauma films are capable of eliciting analogue PTSD symptoms such as intrusions, avoidance symptoms and memory fragmentation (see for a review Holmes and Bourne, 2008). Davies and Clark (1998b) observed that suppression of thoughts of a trauma film resulted in an immediate decrease in intrusions, followed by a rebound when participants were told 
that they no longer had to suppress thoughts about the distressing film. However, in another study, Davies and Clark (1998a) showed that the frequency of intrusions reported during one week after a trauma film could not be predicted by thought suppression. Thus, thought suppression after a trauma film results in an immediate increase in intrusions, but does not have any "long-term" effects one week later. Further studies using trauma films have tested the effect of conceptually-driven processing on analogue PTSD symptoms. In these studies, the effects of conceptually-driven processing were contrasted to data-driven processing, which refers to processing the physical features of the event. The participants who processed the trauma film in a conceptually-driven way reported less intrusions and less self-rated memory fragmentation (Halligan, Clark and Ehlers, 2002; Kindt, van den Hout, Arntz and Drost, 2008). So far, studies have not contrasted the effects of suppression to the supposedly most successful trauma processing style, that is conceptually-driven processing.

In order to test whether thought suppression is detrimental relative to meaningful processing, the current study tested whether conceptually-driven processing immediately after a distressing event is more successful in reducing immediate analogue PTSD symptoms (intrusions, memory fragmentation and state anxiety) than suppression. Conceptually-driven processing and suppression were manipulated in an experimental setting during which healthy participants were shown an extremely aversive film. Participants were assigned to 1) a conceptually-driven processing condition, 2) a suppression condition or 3) a control condition. Four hours later, analogue PTSD symptoms were assessed. These included state anxiety, memory fragmentation and several aspects of intrusions.

\section{Method}

\section{Participants}

Participants were mostly university students, aged 17 to $34(M=22.3, S D=3.6)$. They received $€ 15$ in return for their participation. Individuals were excluded from participation if they had suffered from sexual or physical assault in the past, or if they had already seen the distressing film. Participants were assigned to one of the following conditions: 1) emotional processing condition ( $n=31 ; 25$ females, 6 males); $)$ suppression condition ( $n=29 ; 24$ females, 5 males); 3 ) control condition ( $n=30 ; 24$ females, 6 males).

\section{Stimulus material}

Stimulus material consisted of a 5-minute neutral film and a 29-minute distressing film. The neutral film consisted of landscape images accompanied by restful music. The distressing film was a compilation of "Salò o le 120 giornate di Sodoma" directed by Passolini. It contains scenes showing various kinds of sexual and physical abuse directed at both females and males. The film looks like a documentary and the essence of the story and the chronological order were preserved in the 29-minute compilation. Participants did not realize that they saw only parts of the original. In the first phase, the different characters were introduced. This was followed by sexual and physical violence, which eventually ended in torture. Previous studies have shown that this compilation elicits distress as assessed by subjective (state anxiety) and objective (skin conductance level) measures, dissociation and analogue PTSD symptoms (Kindt and van den Hout, 2003; Kindt, van den Hout and Buck, 2005; Kindt et al., 2008). 


\section{Processing manipulations}

Conceptually-driven processing manipulation. Conceptually-driven processing refers to processing the meaning of the situation and putting it into context. This was manipulated by urging participants to give a rationale for the horrible scenes, to think about the director's intention with the film, and to put the film and the events in the film into context with respect to European history. Participants were provided detailed descriptions of the film in order to facilitate conceptually-driven processing. They were instructed to visualize these descriptions as vividly and in as great a detail as possible. Note that this manipulation can also be regarded as exposure, which is an established treatment for traumatized victims (e.g. Bryant, Moulds, Guthrie, Dang and Nixon, 2003).

After each description, participants were presented with two or three questions about the film. There were 28 questions in total. The questions asked for a rationale of the horrible scenes and what the director aimed to communicate with the scenes. Further questions asked to provide a context for the film with respect to European history. The questions were presented in paper and pencil format. Participants were told that it was very important that they answer all questions.

Suppression manipulation. Suppression of intrusive memories and hindrance of elaborative thoughts was accomplished by occupying the central executive of working memory (Baddeley and Hitch, 1974; Toms, Morris and Ward, 1993). The Random-Interval-Repetition task was used for this purpose (RIR-task; Vandierendonck, De Vooght and van der Goten, 1998). The RIR-task involves responding as fast as possible to two different tones, which are presented at random intervals by pressing one of two buttons. Performing this task leaves less capacity to process intrusive memories or to elaborate.

Participants were instructed to start the Random Interval Repetition task whenever they had thoughts or images about the film, irrespective of whether these thoughts were voluntary or involuntary. Voluntary thoughts refer to elaborative thoughts and involuntary thoughts or images refer to intrusive thoughts. They had to distinguish between a high pitch tone $(1000 \mathrm{~Hz})$ and a low pitch tone $(500 \mathrm{~Hz})$. The tones lasted $100 \mathrm{msec}$. The tones were presented on a computer and the interval was variable $(500$ or $800 \mathrm{msec}$ ). The presentation of the tones and intervals were semi-random in that no more than three subsequent tones had the same pitch or interval. The participant could start the tones by pressing a button. Whenever the task was started it lasted for 30 seconds. Participants were instructed to perform the task during the entire 30 seconds.

Control manipulation. The aim of the control condition was to simulate the natural course of the immediate aftermath of a traumatic event. Traumatized individuals may either think about the event or suppress thoughts about the event by cognitive strategies or by engaging in other activities. Therefore, after the distressing film participants in the control condition were allowed to leave the laboratory during 45 minutes without any instruction.

\section{Manipulation check}

In order to check whether the manipulation was successful, participants were asked to rate the frequency and duration of their elaborative and intrusive thoughts. First, participants were explained the distinction between elaborative and intrusive thoughts by the following instruction: 
In the past 45 minutes you may have thought about the film several times or continuously. Memories can come up as thoughts and/or images about the film. You may have thought back to the film intentionally. You may also have had thoughts or images about the film that came up spontaneously, maybe even against your will. In this case, the thoughts and images intruded. The first two questions are about thoughts and/or images that popped up spontaneously and were intrusive. The following two questions are about intentional thoughts and/or images about the film.

Note that we did not define elaborative thoughts as thoughts about the meaning or context of the film. This was omitted since we did not want to encourage participants in the avoidance condition to start elaborating on the film material in the following 4 hours before assessment of the analogue PTSD symptoms. The questions were as follows: 1) How often during the past 45 minutes did you have intrusive thoughts and/or images? 2) What was the mean duration of these intrusive thoughts and/or images? 3) How often during the past 45 minutes did you think back to the film intentionally? 4) What was the mean duration of intentional thoughts and/or images about the film? Answers to question 1 and 3 were rated on $100 \mathrm{~mm}$ visual analogue scales (VASs), ranging from 0 (not at all) to 100 (very often). Answers to question 2 and 4 were rated on $100 \mathrm{~mm}$ VAS ranging from 0 (very short) to 100 (very long lasting). A higher score refers to more frequent and longer lasting thoughts.

In order to check whether the film was distressing, participants were asked to rate the Spielberger state anxiety scale of the State/Trait Anxiety Inventory (STAI; Spielberger, Gorsuch and Lusthene, 1970). The STAI-state is a commonly used measure of state anxiety and has been shown to have good reliability and validity (Spielberger, Gorsuch, Lushene, Vagg and Jacobs, 1983). Scores ranged from 20-80. A higher score refers to more state anxiety.

\section{Analogue PTSD symptoms}

State Anxiety was also assessed as a dependent variable by the STAI.

Memory fragmentation was assessed subjectively on a $100 \mathrm{~mm}$ VAS. Participants rated the fragmentary quality of their recollections of the film by indicating to what degree their recollections had a snap-shot character from 0 (not at all) to 100 (very much) (see also Kindt and van den Hout, 2003; Kindt et al., 2008).

Intrusive memories were assessed by several aspects of intrusive thoughts and images on separate VASs, that is frequency, duration, vividness, distress and suppression of intrusions during the past 4 hours. Scores ranged from 0 (not at all) to 100 (very often) for frequency, from 0 (very short) to 100 (very long lasting) for duration, from 0 (not at all vivid) to 100 (very vivid) for vividness, from 0 (not at all distressing) to 100 (very distressing) for distress, and from 0 (haven't tried at all to suppress intrusions) to 100 (have tried very often to suppress intrusions) for suppression of intrusions. Thus, higher scores refer to more frequent intrusions, longer-lasting intrusions, more vivid intrusions, more distress caused by the intrusions, and more attempts to suppress intrusions (see also Kindt et al., 2008).

\section{Procedure}

Participants were tested twice: during a morning session and 4 hours later in the afternoon. In the morning session, participants started by watching the neutral film in order to establish a relaxed baseline. They then filled in the STAI, watched the distressing film and then filled in 
the STAI once more in order to test whether the film was indeed distressing. Participants were then assigned to one of the three conditions, thus they processed the film conceptually-driven, suppressed all thoughts and images of the film, or left the laboratory. This manipulation lasted 45 minutes in all three conditions. Immediately after the manipulation, the manipulation check was presented. Questions were asked about frequency and duration of both elaborative and intrusive thoughts.

In the afternoon session, 4 hours later, the dependent variables were assessed: the STAI, several questions referring to memory fragmentation, and several aspects of intrusions. Note that the STAI was administered three times, thus after the baseline film, after the distressing film, and as a dependent variable during the afternoon session. Frequency and duration of intrusions were also assessed after the manipulation in order to check whether the manipulation was successful, and as a dependent variable during the afternoon session. Participants were tested individually.

\section{Statistical analysis}

The assessment of the STAI directly after the neutral film was not normally distributed (Kurtosis $=3.97$; Skewness $=1.03$ ). To verify that the aversive film was distressing, the STAI scores after the neutral and aversive films were compared using the Wilcoxon signed ranks test. Further, using the Kruskal Wallis test, the STAI scores of the three groups after the neutral film were compared. Using the ANOVA, the STAI scores of the three groups after the distressing film were compared between the three groups. Moreover, a paired sample $t$-test was performed to test whether STAI scores assessed 4 hours after the manipulation were still higher than STAI scores assessed after the neutral film. Next, using the ANOVA with Bonferroni correction it was tested whether the manipulation was effective; that is, whether participants in the emotional processing condition reported more frequent and more timeconsuming elaborative and intrusive thoughts than participants in the avoidance condition and whether they differed from participants in the control condition. Finally, using an ANOVA with two planned contrasts, it was tested whether the three conditions differed with respect to the dependent variables. First, an overall group difference will be presented. Then, each time two groups are contrasted. First, the two experimental groups taken together (conceptually-driven and suppression group) were contrasted with the control group. Next, the two experimental groups were contrasted with each other. The weights for the first contrast were: 1 (conceptuallydriven processing group), -2 (control group) and 1 (suppression group). The weights for the second contrast were: 1 (conceptually-driven processing group), 0 (control group) and -1 (suppression group). All tests were two-tailed.

\section{Results}

\section{Manipulation check}

The distressing film was indeed distressing as was shown by an elevated score on the STAI after exposure to the film (mean $=45.8, S D=11.2$ ) as compared to the neutral film (mean $=30.6$, $S D=5.3)(Z=-8.10, p<.001)$. The three groups did not differ in STAI scores after the neutral film $\left(\chi^{2}(2)=1.65, p=.44\right)$, nor after the distressing film $(F(2,87)=.556, p=.58)$. STAI scores assessed 4 hours after the manipulation were still elevated over STAI scores 
Table 1. Means and standard deviations of intrusions and elaborative thoughts in the three conditions immediately after the manipulation

\begin{tabular}{llll}
\hline & \multicolumn{1}{c}{ CPC } & \multicolumn{1}{c}{ SC } & CC \\
\hline Frequency of intrusions & $5.6(3)$ & $4.6(2.9)$ & $4.0(2.7)$ \\
Duration of intrusions & $3.2(2.1)^{\mathrm{a}}$ & $1.9(1.6)^{\mathrm{b}}$ & $2.3(1.7)$ \\
Frequency of elaborative thoughts & $6.8(2.9)^{\mathrm{a}}$ & $4.3(2.6)^{\mathrm{b}}$ & $4.3(2.8)^{\mathrm{b}}$ \\
Duration of elaborative thoughts & $5.0(3.0)^{\mathrm{a}}$ & $3.2(2.6)^{\mathrm{b}}$ & $3.2(2.5)^{\mathrm{b}}$ \\
\hline
\end{tabular}

Within each row, superscripts ${ }^{\mathrm{a}}$ and ${ }^{\mathrm{b}}$ are significantly different from each other $(p<.05)$.

Note: $\mathrm{CPC}=$ conceptually-driven processing condition, $\mathrm{SC}=$ suppression condition, $\mathrm{CC}=$ control condition.

assessed after the baseline film $(t(89)=-2.70, p=.008)$. This implies that the induction of distress was still present when analogue PTSD symptoms were assessed.

To verify that the manipulation regarding conceptually-driven processing and suppression was successful, the three groups were compared regarding frequency and duration of both intrusive and elaborative thoughts. Table 1 presents the means and standard deviations of the intrusive and elaborative thoughts that were assessed immediately after the manipulation, for the three conditions.

With respect to the frequency and duration of intrusive thoughts as assessed immediately after the manipulation, the results show that the three groups did not differ on frequency of intrusions $(F(2,87)=2.48, p=.09)$, but they did differ on duration of intrusive thoughts and images $(F(2.87)=3.83, p=.03)$. That is, participants in the conceptually-driven processing condition reported longer lasting intrusive thoughts than participants in the suppression condition $(t(87)=-2.73, p=.02)$. Comparisons with the control condition were not significant, that is, no differences were found between the conceptually-driven processing condition and the control condition $(t(87)=1.74, p=n . s$.), neither between the suppression condition and control condition $(t(87)=0.99, p=n . s$.$) .$

Regarding the frequency and duration of elaborative thoughts as assessed immediately after the manipulation, the findings show that the groups differed on frequency of elaborative thoughts $(F(2,87)=8.16, p=.001)$, that is, participants in the conceptually-driven processing condition reported more frequent elaborative thoughts than participants in the suppression condition $(t(87)=-3.25, p=.002)$ and in the control condition $(t(87)=3.48, p=.002)$. No difference was found between participants in the suppression condition and the control condition $(t(87)=-.03, p=n . s$. $)$. Finally, the groups also differed on duration of elaborative thoughts $(F(2,87)=4.18, p=.02)$, that is, participants in the conceptually-driven processing condition also reported longer lasting elaborative thoughts than participants in the suppression condition $(t(87)=2.51, p=.04)$ and in the control condition $(t(87)=2.48, p=.046)$. No difference was found between participants in the suppression condition and in the control condition $(t(87)=.06, p=n . s$.$) .$

The manipulation of stimulating elaborative thoughts in the conceptually-driven processing condition as compared to the suppression condition was successful. Even though participants in the conceptually-driven processing condition did not report more intrusions than participants in the suppression condition, they did report longer lasting intrusive thoughts. The RIR-task ended the duration of intrusions but did not decrease the number of intrusions. Therefore, the 
Table 2. Means and standard deviations of dependent variables in the three conditions

\begin{tabular}{lrrr}
\hline & CPC & SC & \multicolumn{1}{c}{ CC } \\
\hline State anxiety & $31.3(6.4)$ & $32.4(7.3)$ & $32.7(7.1)$ \\
Fragmentation & $6.5(2.3)$ & $5.6(2.8)$ & $6.0(2.7)$ \\
Intrusions & & & \\
$\quad$ - Frequency & $2.5(2.0)$ & $2.2(2.1)$ & $2.1(1.8)$ \\
$\quad$ - Duration & $1.6(1.4)$ & $1.5(1.5)$ & $1.6(1.8)$ \\
- Vividness & $4.7(3.4)$ & $4.1(3.1)$ & $4.4(2.8)$ \\
- Distress & $2.3(2.0)$ & $2.7(2.4)$ & $3.0(2.5)$ \\
- Suppression & $3.6(2.8)$ & $3.3(2.9)$ & $3.0(2.9)$ \\
\hline
\end{tabular}

Note: $\mathrm{CPC}=$ conceptually-driven processing condition, $\mathrm{SC}=$ suppression condition, $\mathrm{CC}=$ control condition.

manipulation of suppressing intrusive thoughts and images in the suppression condition as compared to the conceptually-driven processing condition was also regarded as successful.

Effects of manipulating suppression and conceptually-driven processing on analogue PTSD symptoms

No overall differences were found between the three conditions regarding state anxiety $(F(2.87)=.36, p=n . s)$; self-rated fragmentation of the film $(F(2.87)=1.05 ; p=n . s)$; frequency of intrusions $(F(2.87)=.40 ; p=n . s)$, duration of intrusions $(F(2.87)=.10 ; p=n . s)$, vividness of intrusions $(F(2.87)=.28 ; p=n . s)$, distress of intrusions $(F(2.87)=.86 ; p=n . s)$ and suppression of intrusions $(F(2.87)=.30 ; p=n . s)$.

Next, the two experimental groups (conceptually-driven and suppression group) taken together were contrasted with the control group using an ANOVA with two planned contrasts. No differences were found between the two experimental groups, and the control group regarding state anxiety $(t(87)=-.54, p=n . s$. $)$; self-rated fragmentation of the film $(t(87)=$ $.14 ; p=n . s$.$) ; frequency of intrusions (t(87)=.71 ; p=n . s$.$) , duration of intrusions (t(87)=$ $-.38 ; p=n . s$.$) , vividness of intrusions (t(87)=-.003 ; p=n . s$.$) , distress of intrusions$ $(t(87)=-1.03 ; p=n . s$.$) , and suppression of intrusions (t(87)=.69 ; p=n . s$.$) .$

Finally, the two experimental groups were contrasted with each other. Again no differences were found between the conceptually-driven processing group and the suppression group regarding state anxiety $(t(87)=-.65, p=n . s$.$) ; self-rated fragmentation of the film (t(87)=$ $1.44 ; p=n . s$.$) ; frequency of intrusions (t(87)=.54 ; p=n . s$.$) , duration of intrusions (t(87)=$ $.22 ; p=n . s$.$) , vividness of intrusions (t(87)=.74 ; p=n . s$. $)$, distress of intrusions $(t(87)=-.78$; $p=n . s$.$) , and suppression of intrusions (t(87)=.34 ; p=n . s$.). See Table 2 for means and standard deviations of the dependent variables in the three conditions.

\section{Discussion}

The manipulation of conceptually-driven processing and suppression of intrusive thoughts and images was successful, as is evident from the finding that the film-related thoughts of individuals in the conceptually-driven processing condition as compared to the suppression 
condition were longer lasting. Still, the manipulation did not exert the predicted effect on analogue PTSD symptoms. Thus, conceptually-driven processing did not result in fewer symptoms of intrusions, memory fragmentation and anxiety symptoms than suppression.

One might argue that this experimental design is not realistic. Indeed, a distressing film is not traumatic. However, the advantage of an experimental setting over a real trauma setting is that processing style can be manipulated in order to test causality between processing style and analogue PTSD symptoms. Obviously, laboratory studies do not allow conclusions to be drawn about the causality between processing styles and the development of PTSD. Nonetheless, combined with clinical studies, they do contribute to the pathogenesis of PTSD. An experimental design is even more desirable when one of the processing styles under study is thought to be detrimental, as is the case with suppression. Thus the present study allowed us to test the effect of suppression as compared to conceptually-driven processing on analogue PTSD symptoms.

One might further argue that conceptually-driven processing results in an initial increase in distress due to the assignment to visualize the descriptions of the scenes as vividly as possible. This could have masked any beneficial effects of conceptual processing that might have been observed a few days later instead of only a few hours later, as in the present experiment. However, if visualization of the distressing film fragments had increased initial distress, the conceptually-driven processing group should have reported more state anxiety than the control group. This was not observed. Thus, possible beneficial effects of conceptual processing do not appear to be masked by an initial increase in distress. Still, from the present study it can only be concluded that conceptual processing is not favourable over suppression with respect to shortterm symptoms. Future studies may test the effects of suppression versus conceptually-driven processing on analogue PTSD symptoms assessed during one week after the distressing film. The present findings may imply that immediately after a distressing event it may not be crucial to actively process the distressing event. This may, however, only be applicable to aversive stimuli that are not traumatic. The same mechanisms do not necessarily apply to real life traumatic experiences. On the other hand, the present findings are in line with debriefing studies, which showed that forced memory processing immediately after a distressing event is counterproductive (see Emmerik et al., 2002 for a meta-analysis). Early forced elaboration may interfere with successful conceptual processes (see also Ehlers and Clark, 2003).

Furthermore, suppression may have been the coping-style of choice in that participants in the control conditions responded similarly to participants in the suppression condition. They reported less frequent and shorter elaborative thoughts than participants in the conceptuallydriven processing condition. This may indicate that participants in the control condition preferred to suppress rather than dwell on the distressing film. However, this is very speculative since it is only inferred from the reported frequency and duration of elaborative thoughts. We do not know whether these participants purposely suppressed thoughts about the film or whether they were just distracted.

In the present study we assessed suppression by asking participants to perform a task rather than by asking them to suppress the memories of the film. In other words, participants were given a means to suppress their memories of the film. Instructing participants to suppress thoughts and images of the film without providing them with a "distraction-task" may have resulted in different findings. Future studies may compare the effects of this alternative operationalization of suppression to conceptually-driven processing. 
A limitation of the present study is the predominance of female subjects. Further, we concluded that the film was still distressing when analogue symptoms were assessed. However, there is a possibility that this conclusion is not accurate. Baseline state anxiety scores were assessed after participants had watched a neutral film. Watching a neutral film may have reduced state anxiety scores to a level that is lower than normal, and thus increased the chance for a significant difference between pre- and post film distress scores. A further limitation is that the measures used to assess intrusions and memory fragmentation, have not been validated.

Several other studies have tested the relation between suppression and intrusions in trauma victims (Shipherd and Beck, 1999, 2005; Steil and Ehlers, 2000). These studies showed that suppression of trauma related thoughts was related to an increased frequency of these thoughts after the suppression period (rebound effect). However, note that Shipherd and Beck (1999, 2005) observed this rebound effect only in participants with PTSD. This suggests that suppression is mainly detrimental for individuals with PTSD. This may be related to negative interpretation of intrusions. Prospective studies have shown that such negative interpretations are among the best predictors of chronic PTSD (Halligan, Michael, Clark and Ehlers, 2003). When trauma victims interpret their intrusions catastrophically, suppression may be detrimental because the trauma-related beliefs are not corrected by disconfirming information. In the case of negative interpretations, conceptually-driven processing may help victims to restructure these negative interpretations. However, victims without dysfunctional beliefs may have nothing to gain from extensive elaborations. In these individuals, suppression may not be detrimental, but may even help these individuals resuming their lives.

Wegner, Quillian and Houston (1996) tested whether memory fragmentation is caused by suppression. They hypothesized that trauma victims suppress memories of the traumatic event by redirecting their attention to a distracter. Over time, this kind of suppression may result in a memory that is clipped into various frames. As a result, the memory may have become associated with distracters used to suppress these memories, simultaneously dissociating it from the other frames that originally preceded and followed it. They found mixed results regarding objective memory fragmentation after instructed thought suppression about a distressing film. The suppression group scored higher on subjective memory fragmentation as compared to the other two groups (those instructed to think about the film, and those who were given no instruction) combined. This finding was confirmed for suppression of memories of the most traumatic event experienced by students (Rassin, Merckelbach and Muris, 2001). So far researchers have merely focused on the effect of dysfunctional processing styles on memory fragmentation. In the present study, the effect of a dysfunctional processing style (i.e. suppression) on subjective memory fragmentation was contrasted to the effect of a functional processing style (i.e. conceptual processing). The two processing styles did not differ in their effect on subjective memory fragmentation.

The findings of the present study indicate that forced suppression immediately after a traumatic event does not have immediate detrimental effects when compared to conceptual processing. Together with other findings (Seery et al., 2008), it may be suggested that suppression or avoidance is not necessarily a predictor of a bad outcome. Allen (1998) suggests that avoidance of thinking about events has negative effects when individuals who use an avoidant coping style believe that avoidance is harmful (see also McNally et al., 2003). Perhaps avoidance is not detrimental as long as people do not interpret their avoidant coping style negatively. Note that the subjects in the present study were instructed to suppress their thoughts and therefore were unlikely to interpret their suppression attempts as negatively. Future studies may test whether negative meta-cognitions on avoidance mediate the 
relation between an avoidant processing style and post-trauma symptomatology. When testing whether meta-cognitions on avoidance mediate the relation between an avoidant processing style and post-trauma symptomatology one should also control for negative appraisals of intrusions.

In sum, the results of the present study show that, in normal individuals, suppression does not necessarily have detrimental effects shortly after the distressing event as compared to conceptually-driven processing. It may well be that for some trauma victims who are vulnerable for developing chronic PTSD, suppression is highly dysfunctional. That is, individuals who believe that avoidance has detrimental effects, or individuals who interpret their intrusions negatively. Unfortunately, by means of an analogue study it is very difficult (and unethical) to elicit analogue PTSD levels, which are so prominent that individuals start to interpret these analogue symptoms catastrophically. This is especially unfortunate because these misinterpretations may be the distinguishing factor between a possible detrimental or beneficial effect of suppression.

\section{References}

Allen, S. (1998). A qualitative analysis of the process, mediating variables and impact of traumatic childbirth. Journal of Reproductive and Infant Psychology, 16, 107-131.

Baddeley, A. D. and Hitch, G. H. (1974). Working memory. In G. Bower (Ed.), Recent Advances in Learning and Motivation, VIII (pp. 47-90). New York: Academic Press.

Bonanno, G. A., Keltner, D., Holen, A. and Horowitz, M. J. (1995). When avoiding unpleasant emotions might not be such a bad thing: verbal-autonomic response dissociation and midlife conjugal bereavement. Journal of Personality and Social Psychology, 69, 975-989.

Bonanno, G. A., Noll, J. G., Putnam, F. W., O'Neill, M. and Trickett, P. K. (2003). Predicting the willingness to disclose childhood sexual abuse from measures of repressive coping and dissociative tendencies. Child Maltreatment, 8, 302-318.

Bryant, R. A., Moulds, M. L., Guthrie, R. M., Dang, S. T. and Nixon, R. D. (2003). Imaginal exposure alone and imaginal exposure with cognitive restructuring in treatment of posttraumatic stress disorder. Journal of Consulting and Clinical Psychology. 71, 706-712.

Davies, M. I. and Clark, D. M. (1998a). Predictors of analogue post-traumatic intrusive cognitions. Behavioural and Cognitive Psychotherapy, 26, 303-314.

Davies, M. I. and Clark, D. M. (1998b). Thought suppression produces a rebound effect with analogue post-traumatic intrusions. Behaviour Research and Therapy, 36, 571-582.

Ehlers, A. and Clark, D. M. (2000). A cognitive model of posttraumatic stress disorder. Behaviour Research and Therapy, 38, 319-345.

Ehlers, A. and Clark, D. M. (2003). Early psychological interventions for adult survivors of trauma: a review. Biological Psychiatry, 53, 817-826.

Ehring, T., Ehlers, A. and Glucksman, E. (2006). Contribution of cognitive factors to the prediction of posttraumatic stress disorder, phobia and depression after motor vehicle accidents. Behaviour Research and Therapy, 44, 1699-1716.

Emmerik, A. A. P., Kamphuis, J. H., Hulsbosch, A. M. and Emmelkamp, P. M. G. (2002). Single session debriefing after psychological trauma: a meta-analysis. The Lancet, 360, 766-771.

Foa, E. B. and Riggs, D. S. (1993). Posttraumatic stress disorder and rape. In J. Oldman, M . B. Riba and A. Tasman. Annual Review of Psychiatry, Vol. 12 (pp. 273-303). Washington, DC: American Psychiatric Association.

Halligan, S. L., Clark, D. and Ehlers, A. (2002). Cognitive processing, memory, and the development of PTSD symptoms: two experimental analogue studies. Journal of Behavior Therapy and Experimental Psychiatry, 33, 73-89. 
Halligan, S. L., Michael, T., Clark, D. M. and Ehlers, A. (2003). Posttraumatic stress disorder following assault: the role of cognitive processing, trauma memory, and appraisals. Journal of Consulting and Clinical Psychology, 71, 419-431.

Holmes, E. A. and Bourne, C. (2008). Inducing and modulating intrusive emotional memories: a review of the trauma film paradigm. Acta Psychologica, 127, 553-566.

Horowitz, M. J. (1976). Stress Response Syndromes. Northvale, NJ: Aronson.

Kessler, R. C., Sonnega, A., Bromet, E., Hughes, M. and Nelson, C. B. (1995). Posttraumatic stress disorder in the National Comorbidity Survey. Archives of General Psychiatry, 52, 1048-1060.

Kindt, M. and Van Den Hout, M. (2003). Dissociation and memory fragmentation: experimental effects on meta-memory but not on actual memory performance. Behaviour Research and Therapy, 41, 167-178.

Kindt, M., Van Den Hout, M. and Buck, N. (2005). Dissociation related to subjective memory fragmentation and intrusions but not to objective memory disturbances. Journal of Behavior Therapy and Experimental Psychiatry, 36, 43-59.

Kindt, M., Van Den Hout, M., Arntz, A. and Drost, J. (2008). The influence of data-driven versus conceptually-driven processing on the development of PTSD-like symptoms. Journal of Behavior Therapy and Experimental Psychiatry, 39, 546-57.

Mayou, R. A., Ehlers, A. and Hobbs, M. (2000). Psychological debriefing for road traffic accident victims: three-year follow-up of a randomised controlled trial. British Journal of Psychiatry, 176, 589-593.

McNally, R. J., Bryant, R. A. and Ehlers, A. (2003). Does early psychological intervention promote recovery from posttraumatic stress? Psychological Science in the Public Interest, 4, 45-79.

Rassin, E., Merckelbach, H. and Muris, P. (2001). Thought suppression and traumatic intrusions in undergraduate students: a correlational study. Personality and Individual Differences, 31, 485-493.

Rothbaum, B. O., Foa, E. B., Riggs, D. S., Murdock, T. and Walsh, W. (1992). A prospective examination of posttraumatic stress disorder in rape victims. Journal of Traumatic Stress, 5, 455-475.

Seery, M. D., Silver, R. C., Holman, E. A., Ence, W. A. and Chu, T. Q. (2008). Expressing thoughts and feelings following a collective trauma: immediate responses to $9 / 11$ predict negative outcomes in a national sample. Journal of Consulting and Clinical Psychology, 76, 657-667.

Shipherd, J. C. and Beck, J. G. (1999). The effects of suppressing trauma-related thoughts on women with rape-related posttraumatic stress disorder. Behaviour Research and therapy, 37, 99-112.

Shipherd, J. C. and Beck, J. G. (2005). The role of thought suppression in posttraumatic stress disorder. Behavior Therapy, 36, 277-287

Spielberger, C. D., Gorsuch, R. C. and Lushene, R. E. (1970). Manual for the State Trait Anxiety Inventory. Palo Alto, CA: Consulting Psychologists Press.

Spielberger, C. D., Gorsuch, R. L., Lushene, R. E., Vagg, R. E. and Jacobs, G. A. (1983). Manual for the State-Trait Anxiety Inventory. Palo Alto, CA: Consulting Psychologists Press.

Steil, R. and Ehlers, A. (2000). Dysfunctional meaning of posttraumatic intrusions in chronic PTSD. Behaviour Research and Therapy, 38, 537-558.

Stroebe, M. and Stroebe, W. (1991). Does "Grief Work" work? Journal of Consulting and Clinical Psychology, 59, 479-482.

Toms, M., Morris, N. and Ward, D. (1993). Working memory and conditional reasoning. The Quarterly Journal of Experimental Psychology, 46A, 679-699.

Vandierendonck, A., De Vooght, G. and Van Der Goten, K. (1998). Interfering with the central executive by means of a random interval repetition task. The Quarterly Journal of Experimental Psychology, 51A, 197-218.

Wegner, D. M., Quillian, F. and Houston, C. E. (1996). Memories out of order: thought suppression and the disturbance of sequence memory. Journal of Personality and Social Psychology, 71, 680-691. 